\title{
Higgs Boson decay to Tau Pairs at the CMS Experiment
}

\author{
Somnath Choudhury ${ }^{1, a, b}$ \\ ${ }^{1}$ DESY - Hamburg, Germany
}

\begin{abstract}
The results on the standard model Higgs boson in tau pair decay using $25 \mathrm{fb}^{-1}$ of pp collision data at 7 and $8 \mathrm{TeV}$ center-of-mass energies collected by the CMS detector at the LHC has been presented. A direct evidence of the Higgs-lepton coupling is established with the tau pair decay mode. Searches for Higgs bosons decaying to tau leptons in scenarios beyond the standard model such as supersymmetry within the minimal extension of the model has also been reported.
\end{abstract}

\section{Introduction}

The ATLAS and CMS collaborations in July 2012 came out with the observation of a Higgs boson [1, 2] using proton-proton collision data from the LHC at CERN corresponding to integrated luminosities of around $5 \mathrm{fb}^{-1}$ at $7 \mathrm{TeV}$ and $5 \mathrm{fb}^{-1}$ at $8 \mathrm{TeV}$ center-of-mass energies. The LHC completed its first run with the CMS detector having recorded about $95 \%$ of delivered collision data, among which about $90 \%$ were certified and used to obtain the results reported here. The final analysis is based on integrated luminosity of $20 \mathrm{fb}^{-1}$ at $8 \mathrm{TeV}$ in addition to the $5 \mathrm{fb}^{-1}$ at $7 \mathrm{TeV}$ collected by the CMS detector [3]. The observation of a Higgs boson around mass of $125 \mathrm{GeV}$ in the high resolution boson decay channels motivates the search in major fermion decay modes as decays of the Higgs boson to tau pairs and bottom quark pairs have significantly large branching fraction in this mass regime. The $125 \mathrm{GeV}$ Higgs boson opens an interesting avenue for its decays to fermions as this would provide an effective handle for the measurement of the Higgs coupling to fermions. The Higgs boson suffers from quadratically divergent selfenergy corrections at high energies. Numerous extensions to the standard model (SM) have been proposed to address these divergences one of which is supersymmetry, a symmetry between fundamental bosons and fermions, which results in cancellation of the divergences. The minimal supersymmetric extension of the standard model (MSSM), outlays the introduction of 2 Higgs doublets leading to 5 physical Higgs bosons after electroweak symmetry breaking, where, $\mathrm{h}$ and $\mathrm{H}$ are the $\mathrm{CP}$-even scalar bosons, $\mathrm{A}$ is the CP-odd pseudoscalar boson collectively denoted as $\Phi$ and $\mathrm{H}^{+}$and $\mathrm{H}^{-}$are the charged Higgs bosons. If the discovered Higgs boson is the low mass Higgs within MSSM, then the search for its heavy partners gains a lot of interest in the tau pair decay mode which has a branching fraction around $10 \%$ enhanced at all masses.
The CMS detector plays a crucial role in robustly identifying individual particles in the collision events. The detector subsystems employed for the analysis are the pixel detector and the silicon strip tracker forming the innermost component of the detector followed by the electromagnetic calorimeter of lead tungstate crystals and hadronic calorimeter of brass / scintillator samplers which are enveloped by a $3.8 \mathrm{~T}$ superconducting solenoid. Outside the solenoid are the muon chambers which are gas ionization detectors in steel return yoke composed of cathode strip chambers, resistive plates and drift tubes. For the physics object reconstruction in a collision event, CMS uniquely employs a particle flow technique which provides event description in the form of mutually exclusive particles identifying all stable particles produced in the event by combining the capabilities of each sub-detector with the most precise measurement of the energy and direction for each particle and then individual measurements from each sub-detector are combined by a geometrical linking alogorithm providing particle identification on blocks of these linked elements. The experiment had to establish methods to cope with a high number of multiple collisions per beam crossing (pileup), which occurs at high luminosity. The average number of pileup events is about 9 and 21 interactions in the years 2011 and 2012 respectively. The successful mitigation of pileup was demonstrated and an almost uniform response of the missing transverse energy resolution as a function of the number of primary vertices in the event has been achieved. The SM Higgs boson analysis utilises the major Higgs boson production mechanisms in pp collisions in order of decreasing cross-sections namely gluon fusion, vector boson fusion (VBF) and associated production with vector bosons (VH). The MSSM Higgs bosons search utilises the production in gluon fusion and more importantly in association with bottom quarks or in bottom quark fusion to enhance the sensitivity of the Higgs boson coupling to bottom quarks.

\footnotetext{
a for the CMS collaboration

be-mail: somnathchoudhury@gmail.com
} 


\section{SM Higgs to Taus}

The SM $\mathrm{H} \rightarrow \tau \tau$ search [4] is performed using all possible decays in fully leptonic, semi-leptonic and fully hadronic states using the final-state signatures $\mathrm{e} \mu, \mu \mu$, ee, $\mathrm{e} \tau_{h}, \mu \tau_{h}$ and $\tau_{h} \tau_{h}$, where electrons and muons arise from leptonic $\tau$-decays and $\tau_{h}$ denotes hadronic tau lepton decays. To enhance the sensitivity of the search, each of these categories is further divided into three exclusive subcategories according to the nature of the associated jets in the event. The gluon-fusion production mechanism has the largest production cross section. However, in the mass region of interest, background from Drell-Yan production of tau pairs overwhelms the expected Higgs boson signal. This search therefore relies strongly upon the signature of Higgs bosons produced via vector boson fusion (VBF) or in association with a high transverse momentum $\left(\mathrm{p}_{\mathrm{T}}\right)$ jet recoiling against the tau pair. In the former case, the distinct topology of two jets with a large rapidity separation greatly reduces the background. In the latter, requiring a high- $\mathrm{p}_{\mathrm{T}}$ jet both suppresses background, and improves the resolution of the tau-pair invariant mass. The search has been categorized into 0-jet, 1-jet and 2-jet VBF categories to extract the signal which are further split on the basis of hadronic tau $\mathrm{p}_{\mathrm{T}}$ and di-tau $\mathrm{p}_{\mathrm{T}}$ for 0 and 1 jet categories or di-tau $\mathrm{p}_{\mathrm{T}}$, di-jet invariant mass and di-jet pseudorapidity separation for 2 -jet category. The 0 -jet category constrains the background normalization, identification efficiencies and energy scales, the 1-jet category improves the resolution of Higgs boson mass and the VBF category has high signal over background ratio. The analysis is also performed in the $\mathrm{VH}$ category with lepton tagging from vector bosons along with the tau lepton pair.

To distinguish the Higgs boson signal from the background, the tau-pair mass is reconstructed using a maximum likelihood technique. The algorithm estimates the original momentum components of the two taus by maximizing a likelihood with respect to free parameters corresponding to the missing neutrino momenta, subject to kinematic constraints. Other terms in the likelihood take into account the tau-decay phase space and the probability density in the tau transverse momentum, parametrized as a function of the tau-pair mass. This algorithm yields a tau-pair mass with a mean consistent with the true value, and distribution with a nearly Gaussian shape. The relative $\mathrm{m}_{\tau \tau}$ mass resolution estimated from simulation is $10-20 \%$ depending on the di-tau decay channel and category. The likelihood based mass reconstruction allows for a better separation between simulated $125 \mathrm{GeV}$ Higgs signal and $\mathrm{Z} \rightarrow \tau \tau$ background than the visible mass alone, yielding an improvement in the final expected significance of $40 \%$.

In each of these categories, a search is performed for an excess of events in the reconstructed di-tau invariant mass distribution. The largest source of irreducible background is $\mathrm{Z} \rightarrow \tau \tau$ which is estimated using an observed sample of $\mathrm{Z} \rightarrow \mu \mu$ events, where the reconstructed muons are replaced by the reconstructed particles from simulated tau decays, a procedure called "embedding". The normalization for this process is determined from the measurement of the CMS measured cross section.
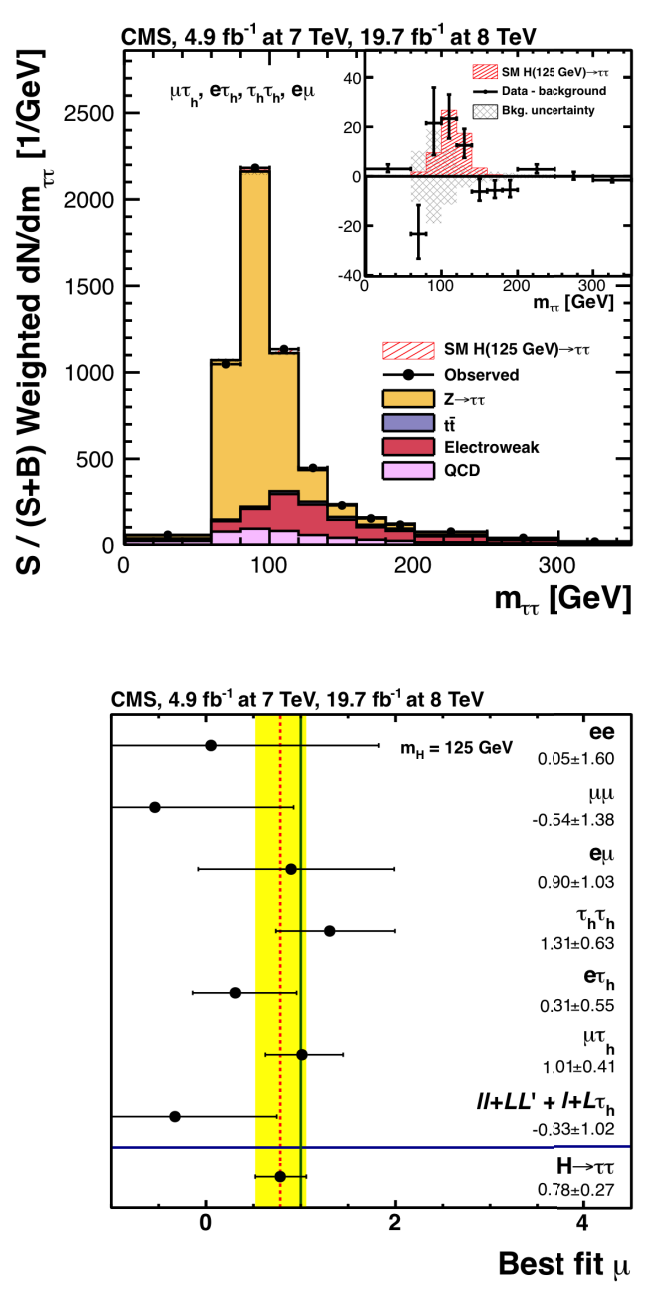

Figure 1. The di-tau invariant mass distribution combining all event categories (top) and the signal strength with respect to SM expectation in different di-tau decay channels and result of the combination (bottom).

The reducible backgrounds $(\mathrm{W}+\mathrm{jets}$, multijet production, $\mathrm{Z}+$ jets) are also evaluated from control samples in data. The QCD multijet background is evaluated using the ratio of opposite-sign (OS) to same-sign (SS) di-tau events and relaxed lepton isolation after an estimate of the $\mathrm{W}+$ jets background using the high transverse mass $\left(\mathrm{m}_{\mathrm{T}}^{\ell E_{\mathrm{T}}^{\text {miss }}}\right)$ sideband $\mathrm{W}$ boson enriched region and extrapolating it to the signal region. The $\mathrm{Z}+$ jets background is evaluated from fake rate and OS/SS ratio with the shape from simulation. The top pair produced events and diboson contribution are estimated using simulation.

Combining all event categories, a broad excess of events is observed in the tau pair invariant mass distribution as seen in Figure 1 over a range of the Higgs boson mass consistent with the $125 \mathrm{GeV}$ scalar boson observed in the high resolution boson decay channels. The observed (expected) significance of the excess at Higgs boson mass of $125 \mathrm{GeV}$ is $3.2 \sigma(3.7 \sigma)$. The best-fit value of the signal strength is $\mu=0.78 \pm 0.27$, obtained in the global fit combining all channels included in this analysis where the $\mathrm{H} \rightarrow \mathrm{WW}$ process has been added as a background for the 

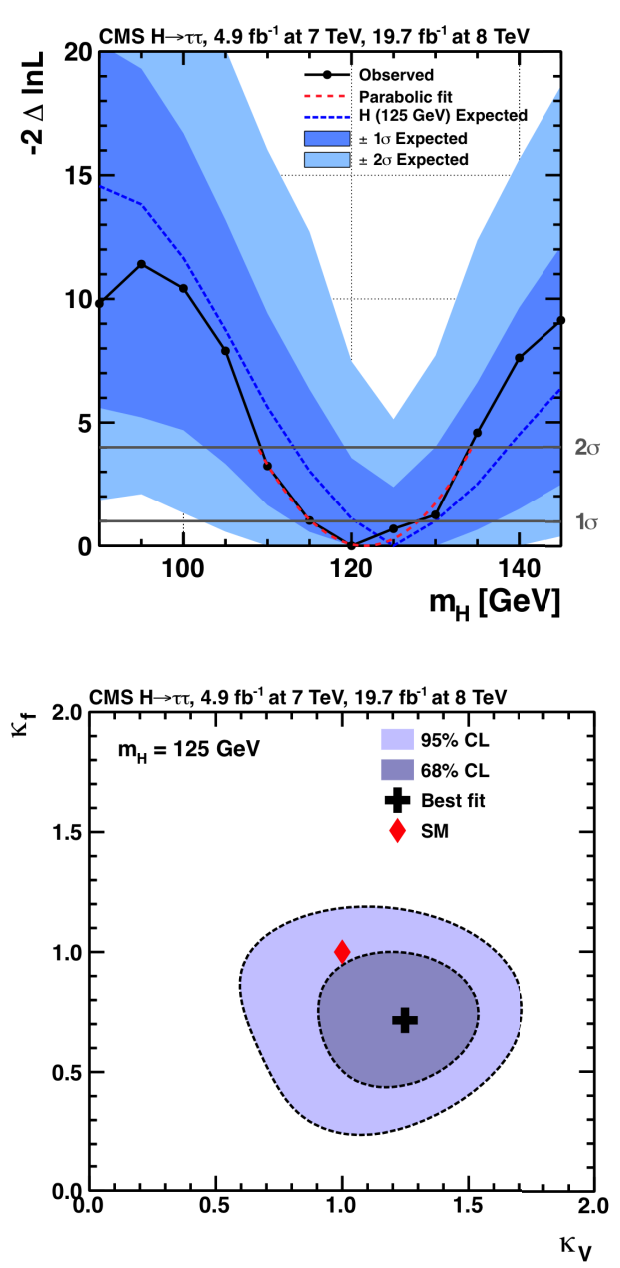

Figure 2. The measured mass from log-likelihood scan in the ditau channel (top) and the 2-D scan of couplings to vector bosons and fermions scaled to the SM expectation (bottom).

observed process. This result provides the first direct indication of the Higgs boson coupling to leptons. The tau pair and bottom quark pair decay modes have been combined at CMS and the significance for the Higgs boson decay to fermions at $125 \mathrm{GeV}$ is $3.8 \sigma$ showing the first direct evidence of Higgs-fermion coupling [5] at the LHC. The mass of the Higgs boson measured in this channel is $122 \pm 7 \mathrm{GeV}$ obtained from a parabolic fit of the loglikelihood scan of the observed mass points in data shown in Figure 2. The measured couplings of the Higgs boson to vector bosons and fermions scaled with respect to SM, shows consistency within around one standard deviations from SM predictions where the $\mathrm{H} \rightarrow \mathrm{WW}$ process has been added as a signal component for this measurement.

\section{MSSM Higgs to Taus}

A Higgs-like state at $125 \mathrm{GeV}$ is rather large for the light MSSM Higgs boson which can be achieved by maximizing the radiative corrections to Higgs mass at 1-loop level. The tau-pair decays of the neutral Higgs bosons in the MSSM, having a branching fraction of roughly $10 \%$, serve as the best experimental signature for this search. The

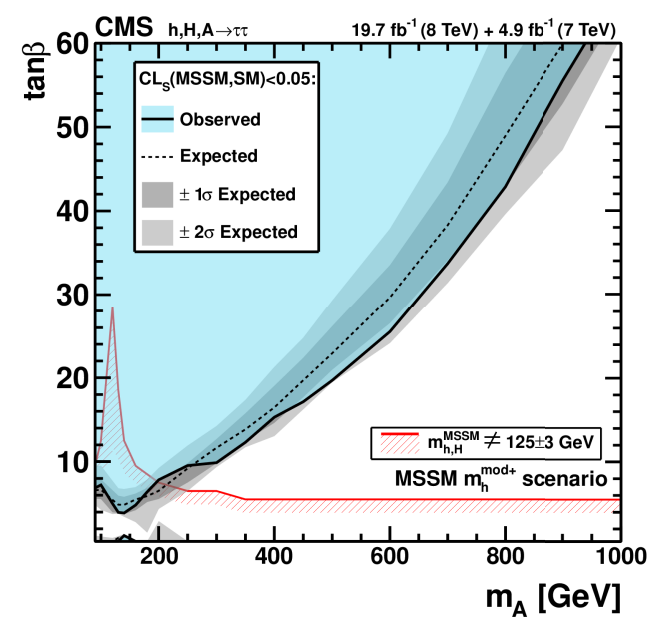

Figure 3. Region in the parameter space of $\tan \beta$ versus $m_{\mathrm{A}}$ excluded at $95 \% \mathrm{CL}$ in the context of a modified mixing $\mathrm{m}_{\mathrm{h}}{ }^{\text {mod }+}$ benchmark scenario. The expected one- and two- standard deviation ranges and the mean expected 95\% CL upper limits are shown together with the observed excluded region.

$\mathrm{b} \overline{\mathrm{b}}$ mode, though it has a much larger branching fraction, suffers from an overwhelming background from multi-jet production. The MSSM Higgs bosons search [6] is performed using the di-tau final-state signatures $\mathrm{e} \mu, \mu \mu, \mathrm{e} \tau_{h}$, $\mu \tau_{h}$ and $\tau_{h} \tau_{h}$. The $\tau_{h}$ decay modes considered for the analysis are a single charged hadron, a charged hadron with neutral pion via the $\rho(770)$ resonance and three charged hadrons via the $a_{1}$ resonance. The data sample has been divided on the b-jet multiplicity into b-tag and non b-tag categories to enhance the sensitivity to bb $\Phi$ coupling:

- b-Tag category: At most one jet with $\mathrm{p}_{\mathrm{T}}>30 \mathrm{GeV}$ and at least one b-tagged jet with $\mathrm{p}_{\mathrm{T}}>20 \mathrm{GeV}$.

- Non b-Tag category: At most one jet with $\mathrm{p}_{\mathrm{T}}>30 \mathrm{GeV}$ and no b-tagged jet with $\mathrm{p}_{\mathrm{T}}>20 \mathrm{GeV}$.

The di-tau invariant mass spectrum shows no evidence for a Higgs boson signal and hence 95\% confidence level (CL) upper bound on the Higgs boson production cross-section times the branching fraction to tau pairs have been set using the mass shape of the tau pair mass spectrum and uncertainties from theory (parton distribution function and renormalization / factorization), normalization (luminosity and efficiency) and shape (energy scale). These limits are further interpreted in the MSSM parameter space on the $\mathrm{m}_{\mathrm{A}}-\tan \beta$ plane as shown in Figure 3 in a modified mixing $\mathrm{m}_{\mathrm{h}}$ benchmark scenario [7] in the stop-top sector with a soft SUSY breaking mass of $1 \mathrm{TeV}$ as the stop mass scale. It excludes all previously unexplored regions reaching as low as $\tan \beta=3.9$ for mass of the pseudoscalar Higgs boson $\mathrm{m}_{\mathrm{A}}=140 \mathrm{GeV}$.

\section{Conclusion}

A broad excess of events is observed for the SM Higgs boson search in the tau pair decay mode consistent with the $125 \mathrm{GeV}$ Higgs boson signal from high resolution boson decay modes $\left(\gamma \gamma\right.$ and $\left.\mathrm{ZZ}^{\star} \rightarrow 4 \ell\right)$ providing the first direct indication of the Higgs boson coupling to leptons. 
Combining tau pair and bottom quark pair decay modes at CMS, the significance for Higgs boson decay to fermions at $125 \mathrm{GeV}$ is $3.8 \sigma$ showing a direct evidence of Higgsfermion coupling at the LHC. The mass of the Higgs boson measured in the di-tau channel is $122 \pm 7 \mathrm{GeV}$ from a parabolic fit of the likelihood scan of the Higgs mass. The search for MSSM Higgs bosons in tau pair decay has set stringent bounds in the $\mathrm{m}_{\mathrm{A}}$ - $\tan \beta$ plane with different MSSM benchmark scenarios tested and reaching as low as $\tan \beta=3.9$ at $m_{\mathrm{A}}=140 \mathrm{GeV}$ at a modified mixing scenario consistent with the observed Higgs boson at $125 \mathrm{GeV}$. In the Run-2 LHC, the measurement of properties of the Higgs-like state in di-tau decay would continue looking for deviations from SM as well as searches for Higgs bosons beyond the SM in tau decays.

\section{References}

[1] The ATLAS Collaboration, Phys. Lett. B 716, 1 (2012).

[2] The CMS Collaboration, Phys. Lett. B 716, 30 (2012).

[3] The CMS Collaboration, JINST 3, S08004 (2008).

[4] The CMS Collaboration, JHEP 05, 104 (2014) and all references therein.

[5] The CMS Collaboration, Nat. Phys. 10, 557 (2014) and all references therein.

[6] The CMS Collaboration, arXiv: 1408.3316 [hep-ex] and all references therein.

[7] M. Carena et. al., Eur.Phys.J. C73, 2552 (2013). 